\section{Veterans who have served in the conflicts of Iraq and Afghanistan are at increased risk of suicide compared to other veterans if they have a psychiatric diagnosis}

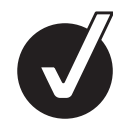

QUESTION

Question: Is there an association between military service in Iraq and Afghanistan, psychiatric EDITOR'S comorbidity and suicide mortality?

CHOICE People: All 5772282 veterans who had used the Veterans Health Administration (VHA) services during fiscal year (FY) 2007 or 2008. Veterans were $90.5 \%$ male and an average age of 60.9 years. Among these veterans, 309108 were Operations Enduring Freedom and Iraqi Freedom (OEF/OIF) veterans who had served in the conflicts of Iraq and Afghanistan and the remaining 5463174 were non-OEF/OIF veterans who had served in other conflicts.

Setting: VHA, USA; 2007-2008.

Risk factors: OEF/OIF status and psychiatric comorbidity. The VHA's National Patient Care Database (NPCD) was used to identify any use of inpatient, residential or outpatient services during FY07 or FY08. Diagnoses of depression, schizophrenia, bipolar disorder, substance use disorder, posttraumatic stress disorder (PTSD) and other anxiety disorders were coded according to ICD-9. VHA users were categorised as OEF/OIF if the date of last deployment in the VHA's OEF/ OIF roster was on or before the end of FY08. Analyses were controlled for sex, age and geographic region. The analysis focused on veterans not currently on active duty.

Outcomes: Suicide death, identified by linking the NPCD to the Centers for Disease Control and Prevention's National Death Index, which coded deaths according to ICD-10 criteria.

\section{METHODS}

Design: Cohort study.
Follow-up period: Approximately 1 year: from first day of FY2008, or first day of use in FY2008 for those not using services in FY2007, to death or last day of FY2008.

\section{MAIN RESULTS}

Compared to non-OEF/OIF veterans, OEF/OIF veterans were on average younger (32.7 vs 62.5 years), included a higher proportion of women (12.5\% vs $9.4 \%$ ), and were more likely to be diagnosed with depression, PTSD and other anxiety disorders. There were 1920 suicide deaths during FY2008, $5 \%$ of which $(n=96)$ were among OEF/OIF veterans. The rate of suicide among OEF/OIF veterans was 38 per 100000 person-years vs 36.0 per 100000 person-years among non-OEF/OIF veterans ( $\mathrm{p}$ value not reported). There was a significant interaction between risk of suicide and being an OEF/OIF veteran and having any psychiatric condition $(p=0.02)$. Among OEF/OIF veterans, any psychiatric condition was associated with a 4.4-fold increase in risk of suicide (HR 4.41, 95\% CI 2.57 to 7.55), compared to a 2.5 -fold increase in risk of suicide among non-OEF/OIF veterans (HR 2.48, 95\% CI 2.27 to 2.71). When looking at specific psychiatric diagnoses, there was significant interaction between OEF/OIF status and depression, schizophrenia, or a substance use disorder. There was no significant effect of OEF/OIF status alone (without psychiatric comorbidity).

\section{CONCLUSIONS}

OEF/OIF veterans are not at an increased risk of suicide mortality compared to non-OEF/OIF veterans, but those with a psychiatric disorder are at almost doubled risk compared to non-OEF/OIF veterans.

\section{ABSTRACTED FROM}

Ilgen MA, McCarthy JF, Ignacio RV, et al. Psychopathology, Iraq and Afghanistan service, and suicide among Veterans Health Administration patients. J Consult Clin Psychol 2012;80:323-30

Correspondence to: Mark A llgen, Department of Psychiatry, University of Michigan, 2800 Plymouth Road, Ann Arbor, Ml 48109, USA; marki@med.umich.edu

Sources of funding: None reported.

- Additional data and references are published online only. To view this file please visit the journal online (http://dx.doi.org/10.1136/eb-2012-100914)
C onsiderable research shows that suicide is a major problem for military veterans and particularly for those who served in Operations Enduring Freedom and Iraqi Freedom (OEF/OIF). In this regard, Ilgen and colleagues report the surprising and discouraging result that OEF/OIF veterans using Veterans Health Administration (VHA) services have suicide rates comparable to those of earlier conflict VHA users (who served during previous wars).

These findings are surprising for at least two reasons. First, OEF/OIF veterans are highly selected volunteers (healthy warriors), whereas earlier conflict veterans were largely conscripts. Second, all recently discharged OEF/OIF veterans are VHA eligible (healthy users) but VHA care is, in effect, restricted to disabled earlier conflict veterans. Thus OEF/OIF veterans should have lower suicide rates than their earlier era counterparts.

How to explain these results? Ilgen and colleagues note that veteran suicide risk is especially high immediately after military separation. Conceivably, then, earlier conflict veterans may have died by suicide prior to the 2007-2008 study period of this study (survivor bias). Nonetheless, consistent with other studies, ${ }^{1}$ "llgen and colleagues show that suicide rates for VHA users of all eras are notably elevated versus the general population.

An additional consideration discussed is the powerful interaction between psychiatric diagnosis and service era. Psychiatric diagnosis, unsurprisingly, elevates the risk of suicide, but especially so for $\mathrm{OEF} / \mathrm{OIF}$ veterans. As noted, efforts must increase to meet behavioural health needs of OEF/OIF veterans.

However, other needs must also be addressed. For example, veterans who die by suicide may not have a documented major psychiatric condition. Analyses by Kaplan et $a^{1}{ }^{1}$ indicate that many veteran suicides were apparently impulsive leg, involving acute alcohol use) and may be responses to life stressors such as job loss or relationship difficulties. Also, female veterans commit suicide at rates much higher than those of civilian women. ${ }^{2}$

The implications are that former service members need additional services. Veterans should at least be provided full healthcare benefits beyond the current 5-year limit, women-focused programmes, employment services and family support.

\section{Mark S Kaplan, ${ }^{1}$ Bentson H McFarland ${ }^{\mathbf{2}}$}

${ }^{1}$ School of Community Health, Portland State University, Portland, Oregon, USA ${ }^{2}$ Department of Psychiatry, Oregon Health \& Science University, Portland, Oregon, USA

\section{Competing interests None.}

